\section{Avaliação da qualidade metodológica de diretrizes de vigilância e manejo clínico de dengue e chikungunya}

\author{
Methodological quality assessment of guidelines \\ for surveillance and clinical management of \\ dengue and chikungunya
}

\author{
Evaluación de la calidad metodológica de
directrices de vigilancia y manejo clínico \\ Evaluación de la calidad metodológica d
directrices de vigilancia y manejo clínico \\ del dengue y chikungunya
} del dengue y chikungunya

\author{
Manuella Carvalho Feitosa 1,2 \\ Pedro Henrique Amparo da Costa Leite 2 \\ Julia Henrique Costa 3 \\ Yara Hahr Marques Hökerberg 3
}

doi: 10.1590/0102-311X00050919

\title{
Resumo
}

A pesquisa objetivou avaliar a qualidade metodológica de diretrizes do Ministério da Saúde brasileiro, da Organização Pan-Americana da Saúde (OPAS) e da Organização Mundial da Saúde (OMS) sobre vigilância e manejo clínico de dengue e chikungunya. Trata-se de um estudo descritivo, no qual a ferramenta Appraisal of Guidelines for Research \& Evaluation Reporting Checklist II (AGREE II) foi aplicada por quatro avaliadores, de forma independente e mascarada, para seis diretrizes. Cada avaliador atribuiu um escore de 1 (discordo totalmente) a 7 (concordo completamente) aos 23 itens dos domínios do AGREE II: escopo e propósito; envolvimento das partes interessadas; rigor no desenvolvimento; clareza da apresentação; aplicabilidade e independência editorial. As diretrizes de dengue da OPAS (média $=5,2$, $D P=0,8)$ e da OMS (média $=4,5, D P=0,5$ ) obtiveram maiores pontuações globais, sendo recomendadas com modificações por todos os avaliadores, e a do Ministério da Saúde (média = 2, 7, DP = 0,4) não foi recomendada por um deles. Já as pontuações das diretrizes de chikungunya foram baixas (médias variando de 2,2 a 3,0) independentemente do órgão que as elaborou. Os dominios com maior conformidade foram "clareza da apresentação" (mediana de 84,7\%) e "escopo e propósito" (77,1\%), e os de menor conformidade foram "independência editorial" (5,2\%) e "rigor no desenvolvimento" (9,1\%). O estudo identificou lacunas na qualidade metodológica das diretrizes relacionadas, principalmente, à transparência nos processos de busca, seleção das evidências científicas e formulação das recomendações, além de falta de clareza quanto ao financiamento e possiveis conflitos de interesses.

Guias de Prática Clínica como Assunto; Avaliação em Saúde;

Infecções por Arbovirus

\author{
Correspondência \\ M. C. Feitosa \\ Centro de Ciências da Saúde, Universidade Federal de Roraima. \\ Av. Capitão Ene Garcez 2413, Boa Vista, RR \\ 69310-000, Brasil. \\ manuella.feitosa@ufrr.br \\ 1 Centro de Ciências da Saúde, Universidade Federal de \\ Roraima, Boa Vista, Brasil. \\ 2 Escola Nacional de Saúde Pública Sergio Arouca, Fundação \\ Oswaldo Cruz, Rio de Janeiro, Brasil. \\ 3 Instituto Nacional de Infectologia Evandro Chagas, Fundação \\ Oswaldo Cruz, Rio de Janeiro, Brasil.
}




\section{Introdução}

As infecções por dengue e chikungunya podem produzir quadros clínicos semelhantes na fase aguda, e durante hiperendemias e epidemias são motivo de intensa procura por atendimento nas unidades de saúde. O diagnóstico precoce, manejo clínico adequado dos pacientes, intervenções de controle vetorial e de monitoramento de casos humanos para a prevenção, detecção e enfrentamento de epidemias são prementes para a diminuição da morbimortalidade por essas arboviroses, o que implica intenso treinamento das equipes de saúde 1,2,3,4. Nesse sentido, as diretrizes clínicas são úteis para orientar as práticas com base em evidências, definir fluxos e otimizar o uso dos recursos disponíveis, considerando-se o custo-benefício das diferentes opções de diagnóstico, tratamento 5 e de medidas de saúde pública que envolvem vigilância epidemiológica e entomológica.

Apesar da dengue ser a mais importante arbovirose que afeta o homem, com vasta distribuição mundial e incidência crescente 6,7 , até a atualidade poucos estudos avaliaram diretrizes de dengue: um avaliou a heterogeneidade de recomendações e uso destes dispositivos 8; outros dois avaliaram a qualidade metodológica de apenas uma diretriz de dengue em meio a outras sobre tópicos diversos 9,10.

De forma semelhante, diretrizes para vigilância e manejo clínico de casos de chikungunya ainda não foram avaliadas de forma crítica, apesar desta doença vir gerando significativos prejuízos econômicos após dezembro de 2013, quando o vírus estabeleceu seu ciclo de transmissão mosquito/ humano nas Américas, com a diminuição da produtividade humana devido à carga da doença crônica incapacitante que é capaz de causar 4,11,12,13.

Em nível internacional, a Organização Mundial da Saúde (OMS) tem elaborado diretrizes para orientar as ações de saúde e para nortear o desenvolvimento de diretrizes nacionais ou regionais. Dentre elas estão a diretriz para diagnóstico, tratamento, prevenção e controle de dengue 14 e aquelas sobre manejo clínico, prevenção e controle da febre de chikungunya 15,16. Por orientar as políticas de saúde, as ações de vigilância epidemiológica e a prática clínica da maioria dos 194 países membros das Nações Unidas, o processo de desenvolvimento desses dispositivos deve ser criterioso e transparente para fornecer recomendações baseadas em uma síntese das melhores evidências disponíveis 9 .

Uma diretriz deve fornecer um resumo baseado em evidências, atual, acessível, transparente e fácil de usar, objetivando melhorar o cuidado. Entretanto, os processos de desenvolvimento de diretrizes variam substancialmente e muitos não atendem os critérios básicos de qualidade, são contraditórios, impraticáveis, inaceitáveis para os usuários ou simplesmente não baseados em evidências $17,18,19,20,21,22$.

Nesse sentido, o Appraisal of Guidelines for Research es Evaluation Reporting Checklist II (AGREE II) é um instrumento baseado em revisões abrangentes da literatura e consenso entre uma equipe internacional ampla de interessados em diretrizes práticas, e é considerada a melhor ferramenta de avaliação da qualidade metodológica de diretrizes disponível. Originalmente publicado em 2003, o instrumento foi refinado, resultando em uma versão mais curta e eficiente com 23 itens sobre o escopo, a população-alvo, os métodos de seleção das evidências, a forma de apresentação e aplicabilidade das recomendações e os conflitos de interesse na elaboração da diretriz, capazes de produzir uma avaliação detalhada 20,23,24,25.

Nessa perspectiva, o presente trabalho objetivou avaliar a qualidade metodológica de diretrizes de vigilância e manejo clínico de dengue e chikungunya.

\section{Metodologia}

Trata-se de um estudo descritivo de avaliação de diretrizes governamentais de acesso livre, dispensando apreciação ética por se restringir a uma análise documental.

Foram incluídas as diretrizes mais recentes sobre vigilância e manejo clínico de dengue e chikungunya elaboradas pelo Ministério da Saúde do Brasil, OMS ou Organização Pan-Americana da Saúde (OPAS), disponíveis no mês de janeiro de 2018 nos sites oficiais destes órgãos, sendo selecionadas as diretrizes da OMS, Dengue: Guidelines for Diagnosis, Treatment, Prevention and Control 14, Guidelines on Clinical Management of Chikungunya Fever 15 e Guidelines for Prevention and Control of Chikungunya 
Fever 16; a diretriz da OPAS Dengue: Guidelines for Patient Care in the Region of the Americas 26; além das do Ministério da Saúde Dengue: Diagnóstico e Manejo Clínico 27 e Chikungunya: Manejo Clínico 28.

Quatro avaliadores (uma médica e uma enfermeira com experiência em vigilância epidemiológica, um economista com experiência na área de incorporação tecnológica em saúde baseada em evidências e uma estudante do último ano de medicina) analisaram de forma independente e mascarada a qualidade metodológica do documento completo das diretrizes. Utilizou-se o AGREE II, que tem 23 itens agrupados em seis domínios: "Escopo e propósito" (3 itens), que diz respeito ao objetivo geral da diretriz, questões de saúde específicas e população-alvo; "Envolvimento das partes interessadas" (3 itens); "Rigor no desenvolvimento" (8 itens), que avalia o processo de busca e síntese das evidências e os critérios adotados para formular as recomendações; "Clareza da apresentação" (3 itens); "Aplicabilidade" (4 itens), que analisa barreiras e facilitadores à implementação da diretriz; e "Independência editorial" ( 2 itens), abrangendo a avaliação de conflitos de interesse e influência de órgãos financiadores. Cada item recebeu um escore de 1 a 7: um indica ausência de informação ou que o conceito é muito mal relatado; dois a seis indicam que a diretriz não atende plenamente os critérios; e sete, que todos os critérios e considerações foram atendidos 17,23,29.

Embora conhecimentos básicos sobre tomada de decisão fundamentada em evidências e assistência médica possam facilitar o uso do AGREE II e a interpretação dos resultados, o manual do usuário permite que indivíduos sem expertise usem o instrumento com confiança de que diretrizes de boa qualidade serão diferenciadas daquelas de baixa 17,18,29. Mesmo assim, optou-se por incluir profissionais de diferentes campos de atuação, experiência profissional e de uso de diretrizes para minimizar o viés de aferição. Os avaliadores estudaram o manual do AGREE II e se reuniram, previamente ao processo de avaliação, para sanar possíveis divergências de entendimento e padronizar os critérios adotados. A avaliação foi feita diretamente no site do AGREE II 30, utilizando-se a ferramenta My AGREE Plus (https://www.agreetrust.org/resource-centre/agree-plus/), que gerava os cálculos das porcentagens de qualidade por domínio com base na soma das pontuações obtidas nos itens do domínio dividida pela pontuação máxima possível para aquele domínio, ambas descontadas da pontuação mínima atribuível ao domínio. As porcentagens de conformidade das diretrizes segundo os itens do AGREE II foram calculadas seguindo-se a mesma lógica. Já a qualidade global das diretrizes foi classificada em uma escala de um (mais baixa) a sete (mais alta), sendo calculada a média e o desvio padrão (DP) da pontuação global dos avaliadores. Além disso, os avaliadores emitiram parecer sobre a recomendação de uso das diretrizes, da seguinte maneira: "sim", "sim com modificações" e "não recomendo".

Os resultados foram apresentados em tabelas com os percentuais de qualidade por domínios e itens do AGREE II, e com comparações das recomendações presentes nas diretrizes.

\section{Resultados}

Seis diretrizes foram avaliadas: três sobre dengue 14,26,27 e três sobre chikungunya 15,16,28. Os percentuais de conformidade estão apresentados na Tabela 1.

Das diretrizes de dengue, a da OPAS 26 obteve a maior pontuação na avaliação global do AGREE II ( édia $=5,2 ; \mathrm{DP}=0,8$ ), seguida pela da OMS 14 (média $=4,5 ; \mathrm{DP}=0,5$ ). Ambas foram recomendadas com modificações pelos avaliadores. A diretriz do Ministério da Saúde 27 (média = 2,7; DP = 0,4) não foi recomendada por um dos avaliadores (Tabela 1).

As diretrizes de chikungunya apresentaram pontuações baixas e semelhantes na avaliação global, com médias variando de 2,2 a 3,0 pontos, sendo a de manejo clínico da OMS 15 a que obteve a pior avaliação geral (média $=2,2$; DP $=0,8$ ) e não foi recomendada por dois avaliadores, resultado que só foi melhor do que o da diretriz brasileira de manejo clínico de chikungunya 28 , da qual três avaliadores não recomendaram o uso.

Com relação aos domínios do AGREE II, as diretrizes de dengue e chikungunya apresentaram melhores escores de conformidade nos domínios "Clareza da apresentação" (mediana = 84,7\%; variação de 72,2 a 97,2\%) e "Escopo e propósito" (mediana = 77,1\%; variação de 63,9 a 93,1\%). As piores avaliações foram nos domínios "Independência editorial" (mediana = 5,2\%; variação de 2,1 a 33,3\%) e "Rigor no desenvolvimento" (mediana = 9,1\%; variação de 4,7 a 43,2\%). 
Tabela 1

Qualidade metodológica das diretrizes internacionais e brasileiras de dengue e chikungunya, segundo o Appraisal of Guidelines for REsearch \& Evaluation Reporting Checklist (AGREE II).

\begin{tabular}{|c|c|c|c|c|c|c|c|}
\hline \multirow{2}{*}{$\begin{array}{l}\text { Domínios do AGREE } \\
\text { II (\%) }\end{array}$} & \multicolumn{6}{|c|}{ Diretrizes } & \multirow{2}{*}{$\begin{array}{l}\text { Mediana } \\
\text { (variação) }\end{array}$} \\
\hline & $\begin{array}{c}\text { Dengue: } \\
\text { Guidelines } \\
\text { for Diagnosis, } \\
\text { Treatment, } \\
\text { Prevention, and } \\
\text { Control (2009) } 14\end{array}$ & $\begin{array}{l}\text { Dengue: } \\
\text { Guidelines for } \\
\text { Patient Care in } \\
\text { the Region of } \\
\text { the Americas } \\
\text { (2016) } 26\end{array}$ & $\begin{array}{c}\text { Dengue: } \\
\text { Diagnóstico e } \\
\text { Manejo Clínico: } \\
\text { Adulto e Criança } \\
\text { (2016) } 27\end{array}$ & $\begin{array}{c}\text { Guidelines } \\
\text { on Clinical } \\
\text { Management } \\
\text { of Chikungunya } \\
\text { Fever (2008) } 15\end{array}$ & $\begin{array}{l}\text { Guidelines for } \\
\text { Prevention } \\
\text { and Control of } \\
\text { Chikungunya } \\
\text { Fever (2009) } 16\end{array}$ & $\begin{array}{l}\text { Chikungunya: } \\
\text { Manejo Clínico } \\
\text { (2017) } 28\end{array}$ & \\
\hline Escopo e propósito & 73,6 & 93,1 & 83,3 & 66,7 & 80,6 & 63,9 & $\begin{array}{c}77,1 \\
(63,9-93,1)\end{array}$ \\
\hline $\begin{array}{l}\text { Envolvimento das partes } \\
\text { interessadas }\end{array}$ & 51,4 & 50 & 31,9 & 44,4 & 27,8 & 23,6 & $\begin{array}{c}38,2 \\
(23,6-51,4)\end{array}$ \\
\hline $\begin{array}{l}\text { Rigor no } \\
\text { desenvolvimento }\end{array}$ & 32,8 & 43,2 & 4,7 & 9,4 & 6,8 & 8,8 & $\begin{array}{c}9,1 \\
(4,7-43,2)\end{array}$ \\
\hline Clareza da apresentação & 86,1 & 97,2 & 88,9 & 72,2 & 76,4 & 83,3 & $\begin{array}{c}84,7 \\
(72,2-97,2)\end{array}$ \\
\hline Aplicabilidade & 38,5 & 46,9 & 14,6 & 4,2 & 19,8 & 12,5 & $\begin{array}{c}17,2 \\
(4,2-46,9)\end{array}$ \\
\hline Independência editorial & 33,3 & 10,4 & 4,2 & 6,2 & 4,2 & 2,1 & $\begin{array}{c}5,2 \\
(2,1-33,3)\end{array}$ \\
\hline $\begin{array}{l}\text { Avaliação global } \\
\text { (média } \pm \text { DP) }\end{array}$ & $4,5 \pm 0,5$ & $5,2 \pm 0,8$ & $2,7 \pm 0,4$ & $2,2 \pm 0,8$ & $3,0 \pm 0,7$ & $2,5 \pm 0,5$ & - \\
\hline $\begin{array}{l}\text { Recomendação } \\
\text { geral de uso }\end{array}$ & $4 \mathrm{RM}$ & $4 \mathrm{RM}$ & 3RM, 1NR & 2RM, 2NR & 3RM, 1NR & 1RM, 3NR & - \\
\hline
\end{tabular}

\%: percentual de conformidade obtido no domínio; DP: desvio padrão da média da avaliação global dos quatro avaliadores; média: média da avaliação global dos quatro avaliadores; RM: recomendada com modificações; NR: não recomendada.

Para a maioria dos domínios do AGREE II, as diretrizes de dengue da OMS 14 e da OPAS 26 obtiveram pontuações mais elevadas do que a brasileira 27 , o que não ocorreu para chikungunya.

O item mais bem-avaliado em todas as diretrizes, item 1 (variação de 87,5 a 100\%) do domínio "Escopo e propósito", refere-se à descrição dos objetivos desses dispositivos (Tabela 2). As três diretrizes de dengue apresentaram conformidade superior a 85\% para os itens 16 (variação de 87,5 a 95,8\%) e 17 (de 91,7 a 100\%), ambos do domínio "clareza da apresentação" e referentes à apresentação das diferentes opções de abordagem da doença e à fácil identificação das recomendações-chave, respectivamente. Todos os itens dos domínios "Escopo e propósito" e "Clareza da apresentação" apresentaram mais de 50\% de conformidade. Dos 23 itens do AGREE II, na diretriz da OPAS 26 60,9\% apresentaram mais de 50\% de conformidade, contra 47,8\% na da OMS 14 e apenas 30,4\% na do Ministério da Saúde 27.

Nenhuma diretriz de chikungunya apresentou $50 \%$ ou mais dos itens com $50 \%$ de conformidade: $30,4 \%$ dos itens obtiveram mais de 50\% de conformidade para a diretriz de prevenção e controle da OMS 16, contra 26,1\% na de manejo clínico 15 e 21,7\% na do Ministério da Saúde 28.

O item 5 (variação de $0 \%$ a 4,2\%), relativo às opiniões e preferências da população-alvo (domínio "Envolvimento das partes interessadas") foi o pior avaliado nas diretrizes de dengue e chikungunya (Tabela 2). Além desse, os itens 9: "Pontos fortes e limitações do conjunto de evidências" (variação de 0\% a 16,7\%), 10: "Métodos para a formulação das recomendações" (variação de 0\% a 25,0\%) e 22: "Possíveis influências do órgão financiador no conteúdo da diretriz" (variação de 4,2\% a 16,7\%) apresentaram conformidade igual ou inferior a $25 \%$ nas seis diretrizes. Os itens 8: "Critérios de seleção de 
Tabela 2

Proporção de conformidade de diretrizes internacionais e brasileiras de dengue e de chikungunya aos itens dos domínios do Appraisal of Guidelines for REsearch \& Evaluation Reporting Checklist (AGREE II).

\begin{tabular}{|c|c|c|c|c|c|c|}
\hline \multirow[t]{2}{*}{ Itens do AGREE II } & \multicolumn{3}{|c|}{ Diretrizes de dengue (\%) } & \multicolumn{3}{|c|}{ Diretrizes de chikungunya (\%) } \\
\hline & $\begin{array}{l}\text { Dengue: Guidelines for } \\
\text { Diagnosis, Treatment, } \\
\text { Prevention, and } \\
\text { Control (2009) } 14\end{array}$ & $\begin{array}{l}\text { Dengue: Guidelines } \\
\text { for Patient Care in } \\
\text { the Region of the } \\
\text { Americas (2016) } 26\end{array}$ & $\begin{array}{c}\text { Dengue: } \\
\text { Diagnóstico e } \\
\text { Manejo Clínico: } \\
\text { Adulto e Criança } \\
\text { (2016) } 27\end{array}$ & $\begin{array}{c}\text { Guidelines } \\
\text { on Clinical } \\
\text { Management of } \\
\text { Chikungunya Fever } \\
\text { (2008) } 15\end{array}$ & $\begin{array}{l}\text { Guidelines for } \\
\text { Prevention } \\
\text { and Control of } \\
\text { Chikungunya Fever } \\
\text { (2009) } 16\end{array}$ & $\begin{array}{l}\text { Chikungunya: } \\
\text { Manejo Clínico } \\
\text { (2017) } 28\end{array}$ \\
\hline \multicolumn{7}{|l|}{$\begin{array}{l}\text { Domínio 1: escopo } \\
\text { e propósito }\end{array}$} \\
\hline 1 & 100,0 & 95,8 & 100,0 & 91,7 & 91,7 & 87,5 \\
\hline 2 & 54,2 & 83,3 & 75,0 & 29,2 & 66,7 & 29,2 \\
\hline 3 & 66,7 & 100,0 & 75,0 & 79,2 & 83,3 & 75,0 \\
\hline \multicolumn{7}{|l|}{$\begin{array}{l}\text { Domínio 2: } \\
\text { envolvimento das } \\
\text { partes }\end{array}$} \\
\hline 4 & 62,5 & 50,0 & 20,8 & 50,0 & 8,3 & 16,7 \\
\hline 5 & 0,0 & 0,0 & 0,0 & 4,2 & 0,0 & 0,0 \\
\hline 6 & 91,7 & 100,0 & 75,0 & 79,2 & 75,0 & 54,2 \\
\hline \multicolumn{7}{|l|}{$\begin{array}{l}\text { 3: rigor do } \\
\text { desenvolvimento }\end{array}$} \\
\hline 7 & 25,0 & 79,2 & 0,0 & 4,2 & 0,0 & 0,0 \\
\hline 8 & 20,8 & 79,2 & 0,0 & 0,0 & 0,0 & 0,0 \\
\hline 9 & 0,0 & 16,7 & 0,0 & 0,0 & 8,3 & 8,3 \\
\hline 10 & 16,7 & 25,0 & 0,0 & 8,3 & 12,5 & 4,2 \\
\hline 11 & 54,2 & 62,5 & 16,7 & 16,7 & 16,7 & 37,5 \\
\hline 12 & 29,2 & 70,8 & 4,2 & 0,0 & 8,3 & 20,8 \\
\hline 13 & 45,8 & 12,5 & 8,3 & 45,8 & 0,0 & 0,0 \\
\hline 14 & 70,8 & 0,0 & 8,3 & 0,0 & 8,3 & 0,0 \\
\hline \multicolumn{7}{|l|}{$\begin{array}{l}\text { Domínio 4: clareza } \\
\text { da apresentação }\end{array}$} \\
\hline 15 & 79,2 & 95,8 & 79,2 & 70,8 & 70,8 & 75,0 \\
\hline 16 & 87,5 & 95,8 & 87,5 & 70,8 & 79,2 & 79,2 \\
\hline 17 & 91,7 & 100,0 & 100,0 & 75,0 & 79,2 & 95,8 \\
\hline \multicolumn{7}{|l|}{$\begin{array}{l}\text { Domínio 5: } \\
\text { aplicabilidade }\end{array}$} \\
\hline 18 & 41,7 & 54,2 & 12,5 & 4,2 & 41,7 & 12,5 \\
\hline 19 & 33,3 & 54,2 & 12,5 & 4,2 & 8,3 & 12,5 \\
\hline 20 & 33,3 & 25,0 & 0,0 & 4,2 & 20,8 & 8,3 \\
\hline 21 & 45,8 & 54,2 & 33,3 & 4,2 & 8,3 & 16,7 \\
\hline \multicolumn{7}{|l|}{$\begin{array}{l}\text { Domínio 6: } \\
\text { independência } \\
\text { editorial }\end{array}$} \\
\hline 22 & 8,3 & 16,7 & 4,2 & 12,5 & 8,3 & 4,2 \\
\hline 23 & 58,3 & 4,2 & 4,2 & 0,0 & 0,0 & 0,0 \\
\hline
\end{tabular}

\%: percentual de conformidade obtido no item. 
evidências" e 23: "Registro e abordagem dos conflitos de interesse" receberam pontuação mínima em todas as diretrizes de chikungunya.

A diretriz de dengue para atendimento ao paciente na região das Américas 26 (115 páginas) foi considerada a mais completa no que diz respeito à presença de recomendações, bem como a citação das evidências de apoio, não contemplando apenas os aspectos relacionados às estratégias de controle de vetores, vigilância entomológica e preparação para emergências, os quais foram abordados na diretriz da OMS 14, com 147 páginas (Tabela 3).

Aspectos clínicos da dengue na criança, por exemplo, estão ausentes na diretriz da OMS 14, e na do Ministério da Saúde 27 (com 58 páginas) são considerados insuficientes e sem ligação com evidências de suporte. Na diretriz da OPAS 26 esses aspectos foram abordados de forma resumida, sendo específicos para recém-nascidos e menores de um ano de idade, mas apresentam indicações de diagnóstico diferencial e tratamento para esta faixa etária, com citação de referências de apoio.

Quadros com diagnóstico diferencial entre dengue, chikungunya e zika estão presentes na diretriz do Ministério da Saúde 27, ausentes na da OMS 14 e apenas a diferenciação entre dengue e chikungunya foi apresentada na diretriz da OPAS 26.

Tabela 3

Recomendações presentes nas diretrizes clínicas de dengue do Ministério da Saúde brasileiro, da Organização Mundial da Saúde (OMS) e da Organização Pan-Americana da Saúde (OPAS)

\begin{tabular}{|c|c|c|c|}
\hline Recomendações & $\begin{array}{c}\text { Dengue: Diagnóstico } \\
\text { e Manejo Clínico } \\
\text { (2016) } 27\end{array}$ & $\begin{array}{c}\text { Dengue: Guidelines } \\
\text { for Patient Care in } \\
\text { the Region of the } \\
\text { Americas (2016) } 26\end{array}$ & $\begin{array}{c}\text { Dengue: Guidelines for } \\
\text { Diagnosis, Treatment, } \\
\text { Prevention, and Control } \\
\text { (2009) } 14\end{array}$ \\
\hline Classificação de casos de dengue (níveis de gravidade) & $+/-$ & +++ & +++ \\
\hline Espectro clínico (fases clínicas) & $+/-$ & +++ & +++ \\
\hline Aspectos clínicos na criança & $+/-$ & +++ & - \\
\hline Aspectos clínicos na gestante & $+/-$ & +++ & - \\
\hline Aspectos clínicos em idosos & - & ++ & - \\
\hline Aspectos clínicos em presença de infecções associadas & - & ++ & - \\
\hline Aspectos clínicos em pacientes hipertensos & $+/-$ & ++ & - \\
\hline $\begin{array}{l}\text { Aspectos clínicos em pacientes com diabetes mellitus, insuficiência } \\
\text { renal, doenças ósseas e articulares. }\end{array}$ & - & ++ & - \\
\hline \multicolumn{4}{|l|}{ Diagnóstico } \\
\hline Diagnóstico laboratorial & $+/-$ & +++ & ++ \\
\hline Diagnóstico diferencial & ++ & ++ & $+/-$ \\
\hline Organização de serviços de laboratório & - & ++ & $+/-$ \\
\hline Serviços Clínicos por nível de complexidade & - & ++ & ++ \\
\hline Classificação de risco (grupos de risco) & $+/-$ & $+/-$ & $+/-$ \\
\hline Manejo de acordo com classificação de risco & $+/-$ & ++ & ++ \\
\hline Tratamento de complicações & $+/-$ & ++ & $+/-$ \\
\hline \multicolumn{4}{|l|}{ Definição de caso } \\
\hline Definição de caso suspeito & $+/-$ & $+/-$ & $+/-$ \\
\hline Definição de caso confirmado & $+/-$ & $+/-$ & - \\
\hline Definição de caso descartado & $+/-$ & $+/-$ & - \\
\hline Organização dos serviços de saúde em surto/epidemia & - & $+/-$ & - \\
\hline Estratégias de controle de vetores & - & - & ++ \\
\hline Vigilância entomológica & - & - & ++ \\
\hline Preparação para emergências e resposta & - & - & ++ \\
\hline Número de páginas & 58 & 115 & 147 \\
\hline
\end{tabular}

+/-: presente, mas sem ligação entre a recomendação e a evidência de suporte; +++: presente e com ligação explícita entre as recomendações e todas as evidências de suporte; ++: presente e com ligação explícita entre as recomendações e algumas evidências de suporte; -: ausente ou insuficiente. 
A maioria das recomendações presentes nas diretrizes de chikungunya não apresenta ligação explícita com evidências da literatura (Tabela 4). A diretriz de manejo clínico da OMS 15 (18 páginas) não apresenta referências, mas apenas uma sugestão de "leitura adicional". Em seu prefácio, consta que ela foi baseada na opinião de especialistas com experiência clínica na Ásia.

A diretriz de chikungunya do Ministério da Saúde 28 (65 páginas), elaborada em um contexto de circulação simultânea de três arbovírus, apresenta um quadro com diagnóstico diferencial entre dengue, chikungunya e zika, aspecto não abordado na diretriz de manejo clínico de chikungunya da OMS 15. A diretriz para a prevenção e controle da OMS 16 (42 páginas) apresenta um quadro de diagnóstico diferencial entre dengue e chikungunya.

\section{Discussão}

As diretrizes mais bem-avaliadas foram sobre dengue, principalmente as internacionais (OPAS 26 e OMS 14), ambas recomendadas com modificações pelos avaliadores. A de dengue do Ministério da Saúde 27, além de obter menor média global do AGREE II, não foi recomendada por um dos avaliadores, que a considerou com qualidade metodológica muito baixa.

Em um estudo que avaliou oito protocolos clínicos de diretrizes terapêuticas do Ministério da Saúde brasileiro, a avaliação da qualidade global apresentou média de 4,2, sendo que dois avaliadores

\section{Tabela 4}

Recomendações presentes nas diretrizes clínicas de chikungunya da Organização Mundial da Saúde e do Ministério da Saúde brasileiro.

\begin{tabular}{|c|c|c|c|}
\hline Recomendações & $\begin{array}{l}\text { Chikungunya: } \\
\text { Manejo Clínico } \\
\text { (2017) } 28\end{array}$ & $\begin{array}{c}\text { Guidelines on Clinical } \\
\text { Management of Chikungunya } \\
\text { Fever (2008) } 15\end{array}$ & $\begin{array}{c}\text { Guidelines for Prevention and } \\
\text { Control of Chikungunya Fever } \\
\text { (2009) } 16\end{array}$ \\
\hline \multicolumn{4}{|l|}{ Diagnóstico } \\
\hline Características clínicas (diagnóstico clínico) & $+/-$ & $+/-$ & +++ \\
\hline Diagnóstico laboratorial & $+/-$ & $+/-$ & $+/-$ \\
\hline Diagnóstico diferencial & ++ & $+/-$ & $+/-$ \\
\hline Manejo clínico na fase aguda & $+/-$ & $+/-$ & $+/-$ \\
\hline Classificação de risco/sinais de gravidade & $+/-$ & $+/-$ & - \\
\hline Aferição da dor & +++ & - & - \\
\hline Abordagem escalonada da dor & ++ & - & - \\
\hline Tratamento farmacológico & ++ & $+/-$ & $+/-$ \\
\hline Tratamento não farmacológico & $+/-$ & $+/-$ & - \\
\hline Manejo clínico na fase subaguda e crônica & $+/-$ & $+/-$ & - \\
\hline Tratamento farmacológico & ++ & - & - \\
\hline Tratamento não farmacológico & $+/-$ & $+/-$ & - \\
\hline Orientações para pacientes pediátricos & $+/-$ & - & - \\
\hline Aferição da dor em crianças & +++ & - & - \\
\hline Manejo da dor da criança & ++ & - & - \\
\hline Manejo de problemas neurológicos & - & $+/-$ & - \\
\hline Manejo de problemas dermatológicos & - & $+/-$ & - \\
\hline Medidas de saúde pública/Ações de vigilância & $+/-$ & $+/-$ & $+/-$ \\
\hline Vigilância, resposta e comunicação de surto & - & - & $+/-$ \\
\hline Definição de caso suspeito/provável & $+/-$ & +++ & +++ \\
\hline Definição de caso confirmado & $+/-$ & +++ & +++ \\
\hline Vigilância e controle vetorial & - & - & ++ \\
\hline Número de páginas & 65 & 18 & 42 \\
\hline
\end{tabular}

+/-: presente, mas sem ligação entre a recomendação e a evidência de suporte; +++: presente e com ligação explícita entre as recomendações e todas as evidências de suporte; ++: presente e com ligação explícita entre as recomendações e algumas evidências de suporte; -: ausente ou insuficiente. 
as recomendaram com modificação e um não recomendou qualquer diretriz 31. Já numa pesquisa que analisou 124 diretrizes atuais da OMS a média do AGREE II foi 5,0, sendo que apenas oito não foram recomendadas 9 . Essa melhor avaliação encontrada para diretrizes internacionais é condizente com outros estudos que avaliaram a qualidade de diretrizes de uma ampla gama de tópicos de saúde, os quais concluíram que organizações supranacionais produziram diretrizes com pontuações do AGREE II significativamente mais altas, sendo recomendadas com mais frequência do que as originárias de um único país 32,33,34.

Uma possível explicação seria a maior disponibilidade de recursos para a elaboração, associada a uma maior fiscalização e cobrança nas etapas do processo de desenvolvimento. Por envolver especialistas de diferentes países e maiores fontes de financiamento, os organismos internacionais provavelmente estão mais bem equipados para elaborar diretrizes mais claras e de maior rigor metodológico 33 .

Vale destacar que a diretriz da OPAS 26 teve como base a diretriz da OMS 14, adaptando-a para a região das Américas, sem alterar as recomendações de tratamento. Apesar de ter incorporado novos aspectos não incluídos na publicação da OMS, o que pode explicar sua melhor qualidade, essa diretriz não descreveu a utilização de uma ferramenta para a adaptação de diretrizes. Essa foi uma das limitações que contribuíram para que ela fosse recomendada com modificações.

Por vez, a diferença de pontuações observada entre diretrizes de âmbitos nacional e internacional não foi observada para as de chikungunya, que apresentaram níveis baixos de conformidade de forma geral. O fato das diretrizes de chikungunya da OMS terem sido publicadas em 200815 e 200916 pode explicar, em parte, a pontuação semelhante do AGREE II entre estas diretrizes e a do Ministério da Saúde 28, elaborada mais recentemente (2017).

Uma avaliação de diretrizes da OMS usando o AGREE II evidenciou que a transparência nos processos de elaboração das diretrizes neste órgão melhorou após críticas de que suas recomendações eram de baixa qualidade, baseadas na opinião de especialistas e raramente usavam métodos sistemáticos baseados em evidências. Isso levou a organização a estabelecer um Comitê de Revisão de Diretrizes em 2007. O estudo demonstrou que houve transição da quase completa ausência de uma seção de métodos para uma descrição bastante completa, com o uso mais amplo de avaliações sistemáticas de evidências e melhor gerenciamento de possíveis conflitos de interesse. No entanto, a maioria das diretrizes publicadas em 2008 e 2009 implementou apenas parcialmente as mudanças, pois já estavam em andamento antes do estabelecimento do comitê 35 . Assim, espera-se que futuras atualizações das diretrizes de chikungunya e dengue da OMS sigam os padrões de desenvolvimento já em vigor e, consequentemente, maior clareza no processo de elaboração desses dispositivos.

Entretanto, como diretrizes elaboradas por organismos internacionais são mais abrangentes, nem sempre adaptadas à realidade de determinados países, as instituições de saúde do Brasil devem identificar as universidades com experiência em diretrizes e trabalhar de forma colaborativa para promover o desenvolvimento e a adaptação de alta qualidade desses dispositivos. Destaca-se, ainda, a importância do sistema de saúde brasileiro e suas diretrizes como referência para outros países da América Latina, Caribe e África 36.

Cerca de 93\% dos 627 médicos, enfermeiros e técnicos de laboratório que participaram de um estudo na América Latina afirmaram ter recebido algum tipo de treinamento em manejo clínico da dengue, baseado nas recomendações de diretrizes clínicas disponíveis, seja no trabalho ou durante a formação profissional 8. Isso demonstra ampla utilização desses documentos no treinamento de equipes de saúde e a importância de que as diretrizes de dengue e chikungunya sejam elaboradas com rigor metodológico, transparência e abordando o contexto peculiar a esse continente, particularmente na atual situação de circulação simultânea de diferentes arbovírus.

A Lei no 12.401 de 201137 reforça que a constituição e alteração de protocolos clínicos e as diretrizes terapêuticas são atribuições do Ministério da Saúde, assessorado pela Comissão Nacional de Incorporação de Tecnologias no SUS (CONITEC). Uma vez que o Departamento de Gestão e Incorporação de Tecnologias em Saúde do Ministério da Saúde preparou uma diretriz metodológica para a elaboração de diretrizes clínicas 38 que estabelece padrões de elaboração e avaliação de qualidade, espera-se que ocorram avanços nas diretrizes organizadas pelo Ministério da Saúde. Entretanto, não foi mencionada qualquer assessoria da CONITEC nos documentos publicados pelo Ministério da Saúde e avaliados neste estudo. 
Vale destacar que a CONITEC disponibilizou para consulta pública uma proposta de escopo de diretriz terapêutica para chikungunya que seria a primeira etapa no processo de elaboração de um protocolo baseado em evidências 39. No entanto, a consulta foi concluída em fevereiro de 2017 e não estão disponíveis no site do órgão informações sobre o que foi feito desde então. Assim, o documento do Ministério da Saúde de 2017 28, avaliado nesta pesquisa, é o que permanece sendo utilizado na abordagem de pacientes com suspeitas de chikungunya em nível nacional.

Em todas as diretrizes avaliadas, os domínios do AGREE II "Escopo e propósito" e "Clareza da apresentação" foram melhor pontuados, resultados condizentes com outros estudos que avaliaram diretrizes de doenças infecciosas do Peru 10, China 40, Índia 41 e publicadas em língua inglesa 42,43. Nesses estudos, as principais recomendações foram facilmente identificáveis nas diretrizes.

Altos escores nesses domínios indicam que as exigências metodológicas em tópicos que auxiliam os profissionais nas suas decisões sobre a utilidade ou não das diretrizes em seus contextos de trabalho estão sendo atendidas. Além disso, o fácil acesso e localização das recomendações contidas nas diretrizes de dengue e chikungunya é primordial, tendo em vista as elevadas demandas de trabalho a que os profissionais estão submetidos em locais de atendimento dos casos suspeitos.

Os piores percentuais do AGREE II para as diretrizes de dengue e chikungunya foram obtidos nos domínios "Rigor no desenvolvimento" e "Independência editorial". Itens que abordam pontos fortes e limitações do conjunto de evidências, métodos para a formulação das recomendações, possíveis influências do órgão financiador e o registro dos conflitos de interesse da equipe de desenvolvedores receberam baixíssimas pontuações. Esses resultados são semelhantes aos de outros estudos que avaliaram diretrizes de doenças infecciosas publicadas na Ásia 40 e América Latina 10. O domínio "Independência editorial" também foi o que recebeu as piores pontuações em estudos com diretrizes sobre doenças hepáticas de uma associação americana 44 e da OMS 9, porém, estes resultados ainda foram melhores do que os obtidos neste estudo.

Deficiências da área da independência editorial podem comprometer a confiabilidade das diretrizes, causando desconfiança dos usuários devido à percepção de possíveis influências decorrentes de conflitos de interesses da equipe e dos órgãos financiadores 25,34 . Entretanto, um estudo da colaboração AGREE mostrou que "Independência editorial" não foi um significativo preditor para recomendações de diretrizes, intenções gerais de uso e qualidade geral 45. Mais estudos são necessários para determinar se os desenvolvedores de diretrizes estão esquecendo de incluir declarações sobre financiamento e conflitos de interesses ou omitindo-as propositalmente por existirem conflitos de interesses que podem ter efeitos negativos no conteúdo das diretrizes 32.

Já a baixa pontuação no "Rigor do desenvolvimento" é particularmente preocupante, pois este domínio pode ser um indicador mais forte de qualidade do que qualquer outro, uma vez que tem efeito mais direto sobre a qualidade das recomendações 32 .

Uma revisão sistemática que incluiu 91 publicações contendo 1.453 diretrizes avaliadas com o AGREE II encontrou que o domínio "Rigor de desenvolvimento" registrou a maior influência nas duas avaliações gerais do AGREE II 46. Semelhantemente, uma pesquisa com 58 autores de publicações sobre avaliações de diretrizes com o AGREE II e usuários de diretrizes de uma rede científica alemã mostrou que os itens de 7 a 12 do domínio "Rigor de desenvolvimento" apresentaram forte influência na recomendação de uso das diretrizes 21 .

Vale destacar que, a pontuação baixa nos itens desse domínio, principalmente nas diretrizes de chikungunya 15,16,28 e na de dengue do Ministério da Saúde 27, esteve relacionada a quase total ausência de descrição dos procedimentos que levaram à seleção de evidências e formulação das recomendações. A falta de uma conexão explícita entre as recomendações contidas nas diretrizes e as evidências que as embasaram pode comprometer a confiabilidade destas recomendações. Melhorias nesse sentido poderiam ser conseguidas por meio da simples inclusão de uma descrição mais detalhada do processo adotado para a seleção das evidências 33 .

Acredita-se, ainda, que existe um vasto conjunto de evidências disponíveis para dengue, por ser uma arbovirose que vem sendo estudada há mais tempo, diferentemente da literatura disponível para chikungunya. No entanto, a falta de evidências não justifica a falta de transparência na formulação das recomendações. Se não existem estudos publicados em determinado aspecto, isso deve ficar claro na diretriz, detalhando-se como as recomendações foram elaboradas nesse caso, o que poderia auxiliar 
a identificar questões prioritárias e estimular uma agenda de pesquisa genuinamente útil para preencher lacunas nos cuidados que tenham um maior impacto na saúde da população 44,47.

Apesar de diferenças consideráveis nas recomendações de diretrizes de dengue usadas em treze países da América Latina e da Ásia sugerirem uma falta de evidências e/ou padrões acordados de gerenciamento de casos de dengue, com grande variação no uso de algoritmos de tratamento, menos de $2 \%$ dos profissionais de saúde pensaram que as orientações destes dispositivos não eram úteis e menos de $1 \%$ discordou das diretrizes 8 . Isso demonstra que os profissionais tendem a confiar na qualidade da informação fornecida por órgãos oficiais, mesmo sem uma avaliação crítica sobre a forma como essas orientações foram formuladas.

Contrapondo essa ideia, autores afirmam que a má formulação de recomendações pode afetar a credibilidade das diretrizes entre os profissionais de saúde e comprometer sua adesão. Além disso, a qualidade metodológica pode ser a diferença entre o sucesso e o fracasso de uma diretriz em alcançar um impacto na saúde, uma vez que os efeitos benéficos só serão obtidos se diretrizes bem desenvolvidas e válidas forem implantadas 10,48 .

$\mathrm{O}$ item pior avaliado no conjunto de diretrizes de dengue e chikungunya foi o que diz respeito às opiniões e preferências da população-alvo (item 5). Outros estudos também não apresentaram uma descrição clara das opiniões e preferências da população-alvo da diretriz $9,34,40,42$. No caso das diretrizes de dengue e chikungunya, que são doenças altamente disseminadas na população, seria importante reconhecer o que os pacientes julgam como importante para ser abordado, com vistas a melhorar os resultados da implantação das diretrizes na perspectiva populacional. Assim, entende-se que existe uma lacuna substancial no envolvimento do paciente no desenvolvimento de diretrizes, sendo que estes podem contribuir na avaliação das prioridades, introduzir novos tópicos, sugerir abordagens holísticas para o atendimento e avaliar como as recomendações interagem com seus valores 49 .

Dentre as possíveis limitações nos resultados desta pesquisa, apontamos que não foi definido previamente como os avaliadores ponderariam os domínios do AGREE II para a avaliação global e recomendação de uso das diretrizes, ficando a critério de cada um fazer o julgamento individual de quais itens/domínios considerariam mais relevantes para esta avaliação geral, sendo uma avaliação completamente subjetiva, conforme orientação do próprio manual do AGREE II.

Entretanto, diversos autores têm sugerido a inclusão de recomendações sobre como considerar os escores de itens e/ou domínios do instrumento para as duas avaliações globais, visando a torná-las mais objetivas, uma vez que sem estas orientações os avaliadores podem acabar ponderando os vários itens/domínios do AGREE II de maneiras distintas, limitando a validade das avaliações gerais $9,21,32,46,50$.

Ressalta-se, como outra possível limitação, que este estudo trata de uma avaliação da qualidade do relato do processo de desenvolvimento de diretrizes, podendo não refletir a qualidade real deste processo. Algumas etapas podem não ter sido relatadas no documento da diretriz ou estão presentes em documentos que não estão facilmente disponíveis para consulta, o que compromete uma avaliação mais fidedigna de sua qualidade metodológica.

O que os desenvolvedores de diretrizes descrevem sobre o seu desenvolvimento pode não fazer justiça a como ela foi realmente desenvolvida 48 . Todavia, as diretrizes devem ser documentos independentes (autônomos), sem a necessidade de contatar os seus desenvolvedores para obter informações adicionais 42 .

Isso reforça a importância da utilidade da ferramenta AGREE II não só para avaliação de diretrizes, mas como instrumento norteador do processo de desenvolvimento e de geração dos relatórios e suplementos de diretrizes, auxiliando na identificação de informações importantes a serem reportadas nestes documentos, tornando o processo mais transparente para os usuários e melhorando a confiabilidade percebida das diretrizes 43 .

Ressalta-se, ainda, que o foco principal do AGREE II está na qualidade do relato dos aspectos metodológicos do desenvolvimento de diretrizes e não na avaliação da qualidade das recomendações subjacentes, uma vez que este tipo de instrumento não pontua a base de evidências do conteúdo das diretrizes. Embora importante, o adequado registro das questões metodológicas é insuficiente para garantir que as recomendações sejam consideradas apropriadas e válidas 18,20,25,51.

Nesse contexto, uma equipe de pesquisadores internacionais está focada no desenvolvimento de uma ferramenta complementar ao AGREE II, o AGREE Recommendation EXcellence (REX), destinado 
a otimizar a implantação, aplicabilidade e qualidade das recomendações de diretrizes 52. Segundo a proposta, o AGREE-REX ajudará os desenvolvedores de diretrizes a criar recomendações clinicamente confiáveis e auxiliará os usuários a avaliar e selecionar aquelas que contenham recomendações confiáveis e apropriadas para a implantação em seus cenários 53.

Outro desafio encontrado neste estudo, com relação ao uso do instrumento AGREE II, diz respeito à sua aplicação a diretrizes que envolvem, além de aspectos clínicos, aspectos voltados à vigilância epidemiológica e organização de serviços de saúde, sendo as diretrizes de arboviroses avaliadas neste estudo consideradas mais amplas e abrangentes que as diretrizes de prática clínica para as quais o instrumento foi projetado. Isso exigiu um esforço dos avaliadores para compreender como alguns itens se aplicariam aos aspectos "não clínicos", uma vez que o manual do usuário do AGREE II não tem exemplos neste âmbito. Por exemplo, a avaliação do domínio "Aplicabilidade” do AGREE II é provavelmente diferente entre diretrizes de prática clínica e de vigilância e, também, entre diretrizes desenvolvidas em nível nacional e diretrizes da OMS, as quais são destinadas a um público global 9. Esses aspectos podem explicar parte da variação das pontuações encontradas nas diretrizes da OMS, OPAS e Ministério da Saúde.

Este estudo traz contribuições ao fazer um diagnóstico da qualidade de diretrizes governamentais de dengue e chikungunya adotadas em níveis nacional e internacional, identificando pontos fortes e fragilidades. Destarte, não foram alvo de avaliação diretrizes e recomendações elaboradas por sociedades de especialidades. A diretriz de dengue da OPAS foi a melhor avaliada, sendo recomendada com modificações. As diretrizes da OMS precisam ser atualizadas, uma vez que as mais recentes foram publicadas no ano de 2009 e novos estudos foram publicados após a dispersão de algumas arboviroses pelo mundo. Já as diretrizes do Ministério da Saúde precisam ser alteradas, passando a ter maior adesão à diretriz de elaboração de diretrizes 38 do próprio Ministério da Saúde. As principais lacunas identificadas estão relacionadas à descrição dos métodos de busca e seleção das evidências para a elaboração das recomendações, o envolvimento do público-alvo, bem como as fontes de financiamento e conflitos de interesses. O aprimoramento da descrição desses aspectos melhoraria a transparência no processo e, portanto, poderia contribuir para melhorar a confiabilidade e adesão de profissionais de saúde e de gestores de saúde às recomendações de manejo clínico e de vigilância epidemiológica nelas contidas.

\section{Colaboradores}

M. C. Feitosa elaborou o projeto de pesquisa, fez as buscas das diretrizes, avaliou as diretrizes, interpretou os resultados das avaliações, elaborou a redação da primeira versão do artigo, revisou e aprovou a versão final. P. H. A. C. Leite avaliou as diretrizes, organizou os dados das avaliações para análise, interpretou os resultados das avaliações, revisou a versão final do artigo, formatou as referências e tabelas, revisou e aprovou a versão final. J. H. Costa avaliou as diretrizes, interpretou os resultados das avaliações, revisou criticamente o artigo e aprovou a versão final. Y. H. M. Hökerberg orientou a elaboração do projeto de pesquisa, fez as buscas das diretrizes, avaliou as diretrizes, interpretou os resultados das avaliações, revisou criticamente a primeira versão do artigo e aprovou a versão final.

\section{Informações adicionais}

ORCID: Manuella Carvalho Feitosa (0000-00018736-4525); Pedro Henrique Amparo da Costa Leite (0000-0003-4919-8831); Julia Henrique Costa (0000-0002-6949-7938); Yara Hahr Marques Hökerberg (0000-0001-7140-7172).

\section{Agradecimentos}

M. C. Feitosa e P. H. A. C. Leite receberam bolsa de doutorado da Escola Nacional de Saúde Pública Sergio Arouca, Fundação Oswaldo Cruz. 


\section{Referências}

1. Donalisio MR, Freitas ARR. Chikungunya in Brazil: an emerging challenge. Rev Bras Epidemiol 2015; 18:283-5.

2. Fathima AS, Manimegalai D, Hundewale N. A review of data mining classification techniques applied for diagnosis and prognosis of the arbovirus-dengue. International Journal of Computer Science Issues 2011; 8:322-8.

3. Muller DA, Depelsenaire ACI, Young PR. Clinical and laboratory diagnosis of dengue virus infection. J Infect Dis 2017; 215 Suppl 2:S8995.

4. Weaver SC. Arrival of chikungunya virus in the new world: prospects for spread and impact on public health. PLoS Negl Trop Dis 2014; 8:e2921.

5. Institute of Medicine; Graham R, Mancher M, Miller Wolman D, Greenfield S, Steinberg E. Clinical practice guidelines we can trust. Washington DC: The National Academies Press; 2011.

6. Messina JP, Brady OJ, Scott TW, Zou C, Pigott DM, Duda KA, et al. Global spread of dengue virus types: mapping the 70 year history. Trends Microbiol 2014; 22:138-46.

7. Stanaway JD, Shepard DS, Undurraga EA, Halasa YA, Coffeng LE, Brady OJ, et al. The global burden of dengue: an analysis from the Global Burden of Disease Study 2013. Lancet Infect Dis 2016; 16:712-23.

8. Santamaria R, Martinez E, Kratochwill S, Soria C, Tan LH, Nuñez A, et al. Comparison and critical appraisal of dengue clinical guidelines and their use in Asia and Latin America. Int Health 2009; 1:133-40.

9. Burda BU, Chambers AR, Johnson JC. Appraisal of guidelines developed by the World Health Organization. Public Health 2014; 128:444-74.

10. Canelo-Aybar C, Balbin G, Perez-Gomez A, Florez ID. Guías de práctica clínica en el Perú: evaluación de su calidad usando el instrumento AGREE II. Rev Peru Med Exp Salud Pública 2016; 33:732-8.

11. Caglioti C, Lalle E, Castilletti C, Carletti F, Capobianchi MR, Bordi L. Chikungunya virus infection: an overview. New Microbiol 2013; 36:211-27.

12. Mathew AJ, Ganapati A, Kabeerdoss J, Nair A, Gupta N, Chebbi P, et al. Chikungunya Infection: a global public health menace. Curr Allergy Asthma Rep 2017; 17:13.

13. Silva LA, Dermody TS. Chikungunya virus: epidemiology, replication, disease mechanisms, and prospective intervention strategies. J Clin Invest 2017; 127:737-49.

14. Special Programme for Research and Training in Tropical Diseases, World Health Organization. Dengue: guidelines for diagnosis, treatment, prevention and control. Geneva: World Health Organization; 2009.

15. World Health Organization. Guidelines on clinical management of chikungunya fever. New Delhi: WHO Regional Office for SouthEast Asia; 2008.
16. WHO Regional Office for South-East Asia. Guidelines for prevention and control of chikungunya fever. New Delhi: WHO Regional Office for South-East Asia; 2009.

17. Brouwers MC, Kho ME, Browman GP, Burgers JS, Cluzeau F, Feder G, et al. AGREE II: advancing guideline development, reporting and evaluation in health care. Can Med Assoc J 2010; 182:E839-42.

18. Burls A. AGREE II-improving the quality of clinical care. Lancet 2010; 376:1128-9.

19. Dans AL, Dans LF. Appraising a tool for guideline appraisal (the AGREE II instrument). J Clin Epidemiol 2010; 63:1281-2.

20. Eikermann M, Holzmann N, Siering U, Rüther A. Tools for assessing the content of guidelines are needed to enable their effective use - a systematic comparison. BMC Res Notes 2014; $7: 853$.

21. Hoffmann-Eßer W, Siering U, Neugebauer EAM, Brockhaus AC, McGauran N, Eikermann M. Guideline appraisal with AGREE II: online survey of the potential influence of AGREE II items on overall assessment of guideline quality and recommendation for use. BMC Health Serv Res 2018; 18:143.

22. Qaseem A, Forland F, Macbeth F, Ollenschläger $G$, Phillips S, van der Wees $P$, et al. Guidelines International Network: toward international standards for clinical practice guidelines. Ann Intern Med 2012; 156:525-31.

23. AGREE Collaboration. Development and validation of an international appraisal instrument for assessing the quality of clinical practice guidelines: the AGREE project. Qual Saf Health Care 2003; 12:18-23.

24. Brouwers MC, Kerkvliet K, Spithoff K, AGREE Next Steps Consortium. The AGREE Reporting Checklist: a tool to improve reporting of clinical practice guidelines. BMJ 2016; 352:i1152.

25. Siering U, Eikermann M, Hausner E, Hoffmann-Eßer W, Neugebauer EA. Appraisal tools for clinical practice guidelines: a systematic review. PLoS One 2013; 8:e82915.

26. Pan American Health Organization. Dengue: guidelines for patient care in the region of the Americas. 2nd Ed. Washington DC: Pan American Health Organization; 2016.

27. Diretoria Técnica de Gestão, Secretaria de Vigilância em Saúde, Ministério da Saúde. Dengue: diagnóstico e manejo clínico: adulto e criança. 5a Ed. Brasília: Ministério da Saúde; 2016.

28. Departamento de Vigilância das Doenças Transmissíveis, Secretaria de Vigilância em Saúde, Ministério da Saúde. Chikungunya: manejo clínico. Brasília: Ministério da Saúde; 2017.

29. Brouwers MC, Kho ME, Browman GP, Burgers JS, Cluzeau F, Feder G, et al. Development of the AGREE II, part 2: assessment of validity of items and tools to support application. Can Med Assoc J 2010; 182:E472-8. 
30. AGREE Collaboration. AGREE: Advancing the science of practice guidelines. https://www. agreetrust.org/ (acessado em 08/Fev/2018).

31. Ronsoni RM, Pereira CCA, Stein AT, Osanai $\mathrm{MH}$, Machado CJ, Ronsoni RDM, et al. Avaliação de oito Protocolos Clínicos e Diretrizes Terapêuticas (PCDT) do Ministério da Saúde por meio do instrumento AGREE II: um estudo piloto. Cad Saúde Pública 2015; 31:115762.

32. Alonso-Coello P, Irfan A, Solà I, Gich I, Delgado-Noguera $M$, Rigau $D$, et al. The quality of clinical practice guidelines over the last two decades: a systematic review of guideline appraisal studies. Qual Saf Health Care 2010; 19:e58.

33. Armstrong JJ, Goldfarb AM, Instrum RS, MacDermid JC. Improvement evident but still necessary in clinical practice guideline quality: a systematic review. J Clin Epidemiol 2017; 81:13-21.

34. Agbata EN, Padilla PF, Agbata IN, Armas LH, Solà I, Pottie K, et al. Migrant healthcare guidelines: a systematic quality assessment. J Immigr Minor Health 2019; 21:401-3.

35. Sinclair D, Isba R, Kredo T, Zani B, Smith H, Garner P. World Health Organization guideline development: an evaluation. PLoS One 2013; 8:e63715.

36. Molino CGRC, Romano-Lieber NS, Ribeiro E, de Melo DO. Non-communicable disease clinical practice guidelines in Brazil: a systematic assessment of methodological quality and transparency. PLoS One 2016; 11:e0166367.

37. Brasil. Lei no 12.401, de 28 de abril de 2011. Altera a Lei no 8.080, de 19 de setembro de 1990, para dispor sobre a assistência terapêutica e a incorporação de tecnologia em saúde no âmbito do Sistema Único de Saúde - SUS. Diário Oficial da União 2011; 29 abr.

38. Departamento de Gestão e Incorporação de Tecnologias em Saúde, Secretaria de Ciência, Tecnologia e Insumos Estratégicos, Ministério da Saúde. Diretrizes metodológicas: elaboração de diretrizes clínicas. Brasília: Ministério da Saúde; 2016.

39. Comissão Nacional de Incorporação de Tecnologia no SUS. Proposta de protocolo clínico e diretrizes terapêuticas para chikungunya: escopo. Brasília: Comissão Nacional de Incorporação de Tecnologias no SUS; 2017.

40. Wu D, Jiang W, Yu L, Wang Y, Tao Y, Tang H, et al. Quality assessment of clinical practice guidelines for infectious diseases in China. J Evid Based Med 2018; 11:95-100.

41. Bhaumik S, Jagadesh S, Ellatar M, Kohli N, Riedha M, Moi M. Clinical practice guidelines in India: Quality appraisal and the use of evidence in their development. J Evid Based Med 2018; 11:26-39.

42. Lytvyn L, Mertz D, Sadeghirad B, Alaklobi F, Selva A, Alonso-Coello P, et al. Prevention of clostridium difficile infection: a systematic survey of clinical practice guidelines. Infect Control Hosp Epidemiol 2016; 37:901-8.
43. Dickson C, Arnason T, Friedman DS, Metz G, Grimshaw JM. A systematic review and appraisal of the quality of practice guidelines for the management of Neisseria gonorrhoeae infections. Sex Transm Infect 2017; 93:487-92.

44. Koh C, Zhao X, Samala N, Sakiani S, Liang TJ, Talwalkar JA. AASLD clinical practice guidelines: a critical review of scientific evidence and evolving recommendations. Hepatology 2013; 58:2142-52.

45. Brouwers MC, Kho ME, Browman GP, Burgers JS, Cluzeau F, Feder G, et al. Development of the AGREE II, part 1: performance, usefulness and areas for improvement. Can Med Assoc J 2010; 182:1045-52.

46. Hoffmann-Eßer W, Siering U, Neugebauer EAM, Brockhaus AC, Lampert U, Eikermann M. Guideline appraisal with AGREE II: systematic review of the current evidence on how users handle the 2 overall assessments. PLoS One 2017; 12:e0174831.

47. Kliner M, Poole K, Sinclair D, Garner P. Preventing malaria in international travellers: an evaluation of published English-language guidelines. BMC Public Health 2014; 14:1129.

48. Graham ID, Calder LA, Hébert PC, Carter AO, Tetroe JM. A comparison of clinical practice guideline appraisal instruments. Int $\mathrm{J}$ Technol Assess Health Care 2000; 16:1024-38.

49. Armstrong MJ, Bloom JA. Patient involvement in guidelines is poor five years after institute of medicine standards: review of guideline methodologies. Res Involv Engagem 2017; 3:19.

50. Hoffmann-Eßer W, Siering U, Neugebauer EAM, Lampert U, Eikermann M. Systematic review of current guideline appraisals performed with the Appraisal of Guidelines for Research \& Evaluation II instrument-a third of AGREE II users apply a cut-off for guideline quality. J Clin Epidemiol 2018; 95:120-7.

51. Vlayen J, Aertgeerts B, Hannes K, Sermeus W, Ramaekers D. A systematic review of appraisal tools for clinical practice guidelines: multiple similarities and one common deficit. Int J Qual Health Care 2005; 17:235-42.

52. Makarski J, Brouwers MC; AGREE Enterprise. The AGREE Enterprise: a decade of advancing clinical practice guidelines. Implement Sci 2014; 9:103.

53. Brouwers MC, Spithoff K, Kerkvliet K, Florez ID. Development and validity testing of the AGREE-REX, a tool to evaluate the clinical credibility and implementability of clinical practice guideline recommendations. https:// abstracts.cochrane.org/2017-global-evidencesummit/development-and-validity-testingagree-rex-tool-evaluate-clinical (acessado em 08/Fev/2018). 
Abstract

The study aimed to assess the methodological quality of guidelines by the Brazilian Ministry of Health, Pan American Health Organization (PAHO), and World Health Organization (WHO) on surveillance and clinical management of dengue and chikungunya. This was a descriptive study in which the tool Appraisal of Guidelines for Research \& Evaluation Reporting Checklist II (AGREE II) was applied by four evaluators in independent and masked fashion for six guidelines. Each evaluator assigned a score from 1 (disagree completely) to 7 (agree completely) to the 23 items in the AGREE II domains: scope and purpose; stakeholder involvement; rigor in the development; clarity of presentation; applicability; and editorial independence. The dengue guidelines by PAHO (mean $=5.2, S D=0.8)$ and $W H O($ mean $=$ 4.5, $S D=0.5)$ obtained the highest overall scores and were recommended with modifications by all the evaluators, while the Brazilian Ministry of Health guidelines (mean $=2.7, S D=0.4)$ were not recommended by any of them. Meanwhile, the chikungunya guidelines scored low (means from 2.2 to 3.0) for all three agencies. The domains with the greatest conformity were "clarity of presentation" (median 84.7\%) and "scope and purpose" (77.1\%), while those with the lowest conformity were "editorial independence" (5.2\%) and "rigor in development" (9.1\%). The study identified gaps in the guidelines' methodological quality, mainly in transparency of the work processes, selection of scientific evidence, and formulation of recommendations, besides lack of clarity in financing and possible conflicts of interest.

Practice Guidelines as Topic; Health Evaluation; Arbovirus Infections

\section{Resumen}

El objetivo de la investigación fue evaluar la calidad metodológica de las directrices del Ministerio de Salud (MS) brasileño, de la Organización Panamericana de la Salud (OPAS) y de la Organización Mundial de la Salud (OMS) sobre vigilancia y manejo clínico del dengue y chikungunya. Se trata de un estudio descriptivo, en el cual la herramienta Appraisal of Guidelines for Research \& Evaluation Reporting Checklist II (AGREE II) fue aplicada por parte de cuatro evaluadores, de forma independiente y oculta, en seis directrices. Cada evaluador atribuyó una puntuación de 1 (en desacuerdo totalmente) a 7 (concuerdo completamente) a los 23 items de los dominios del AGREE II: alcance y propósito; implicando las partes interesadas; rigor en el desarrollo; claridad de la presentación; aplicabilidad e independencia editorial. Las directrices del dengue de la OPAS (media $=$ $5,2, D P=0,8)$ y $O M S$ (media $=4,5, D P=0,5) o b$ tuvieron mayores puntuaciones globales, siendo recomendadas con modificaciones por todos los evaluadores, respecto a las del Ministerio de Salud (media $=2,7, D P=0,4)$ no se recomendó por parte de uno de ellos. Ya las puntuaciones de las directrices de chikungunya fueron bajas (medias variando de 2,2 a 3,0), independientemente del órgano que las elaboró. Los dominios con mayor conformidad fueron "clareza de la presentación" (media de $84,7 \%)$ y "alcance y propósito" (77,1\%), mientras que los de menor conformidad fueron "independencia editorial" $(5,2 \%)$ y "rigor en el desarrollo" (9,1\%). El estudio identificó lagunas en la calidad metodológica de las directrices relacionadas, principalmente, respecto a la transparencia en los procesos de búsqueda, selección de las evidencias científicas y formulación de las recomendaciones, además de la falta de claridad respecto a la financiación y posibles conflictos de intereses.

Guias de Práctica Clínica Como Asunto; Evaluación en Salud; Infecciones por Arbovirus
Recebido em 14/Mar/2019

Versão final reapresentada em 02/Nov/2019

Aprovado em 10/Jan/2020 